\title{
More evidence of localization in the low-lying Dirac spectrum
}

\section{Bernard}

Physics Department, Washington University, St. Louis, MO 63130, USA

E-mail: cb@lump.wustl.edu

\section{Ph. de Forcrand}

Institute for Theoretical Physics, ETH Zürich, CH-8093 Zürich, Switzerland

CERN, Theory Division, CH-1211 Geneva 23, Switzerland,

E-mail: forcrandephys.ethz.ch

\section{Steven Gottlieb and L. Levkova}

Physics Department, Indiana University, Bloomington, IN 47405, USA

E-mail: sgeindiana.edu llevkova@indiana.edu.

\section{U. M. Heller}

American Physical Society, One Research Road, Box 9000, Ridge, NY 11961-9000, USA

E-mail: hellereaps.org

\section{J. E. Hetrick*}

Physics Department, University of the Pacific, Stockton, CA 95211, USA

E-mail: jhetrick@pacific.edu

\section{O. Jahn}

Center for Theoretical Physics, MIT, Cambridge, MA 02139, USA

E-mail: jahn@mit.edu

\section{F. Maresca}

Physics Department, University of Utah, Salt Lake City, UT 84112, USA

E-mail: maresca@physics.utah.edu

\section{B. Renner and D. Toussaint}

Physics Department, University of Arizona, Tucson, AZ 85721, USA

E-mail: dru@physics.arizona.edu, doug@physics.arizona.edu

\section{R. Sugar}

Physics Department, University of California, Santa Barbara, CA 93106, USA

E-mail: sugar@physics.ucsb.edu

We have extended our computation of the inverse participation ratio of low-lying (asqtad) Dirac eigenvectors in quenched SU(3). The scaling dimension of the confining manifold is clearer and very near 3 . We have also computed the 2-point correlator which further characterizes the localization.

XXIIIrd International Symposium on Lattice Field Theory

25-30 July 2005

Trinity College, Dublin, Ireland

\footnotetext{
* Speaker.
} 


\section{Introduction}

The study of the low-lying eigenmodes of the Dirac operator (LDE), those corresponding to the smallest eigenvalues, has a rich history since these modes are thought to be representative of, if not responsible for, much of the infrared behavior in QCD. Such effects include

- Chiral Symmetry Breaking à la Banks and Casher where $\langle\bar{\psi} \psi\rangle \sim \rho_{\lambda}(0)$

- The low eigenmodes of $\not \supset$ dominate quark propagators

- Confinement in many scenarios is thought to be related to topological excitations: instantons, monopoles, or vortices. These objects all localize Dirac zero-modes in some way.

Thus we are interested to learn what we can about this localization, if indeed it exists, and then to characterize it in some quantitative way. Hopefully this characterization can tell us something about the mechanisms responsible for localization.

\section{Inverse Participation Ratio}

The Inverse Participation Ratio (IPR) provides a quantitative number which characterizes the localization of a scalar field. For the LDEs, it is defined as

$$
I_{i}=V \sum_{x} \rho_{i}^{2}(x)
$$

where $V$ is the number of lattice sites $x$, and $i$ labels which eigenmode is under consideration,

$$
\rho_{i}(x)=\psi_{i}^{\dagger} \psi_{i}(x) .
$$

$\psi_{i}(x)$ is the $i$-th lowest eigenvector of the (asqtad) Dirac operator and

$$
\sum_{x} \rho_{i}(x)=1
$$

The IPR takes the following values in cases of different localization:

$$
\begin{aligned}
& I=1 \quad \text { if } \rho \text { is constant, } \\
& I=1 / f \text { if } \rho \text { is localized (and constant) on a fraction } f \text { of sites, and } \\
& I=V \quad \text { if } \rho=\delta_{x, x_{0}} .
\end{aligned}
$$

As was first pointed out in [1], the fraction of points involved in localization should scale with the dimension of the localizing manifold. For example,

$$
f_{1-\operatorname{dim}}=\frac{\mathscr{L} / a}{V / a^{4}} \quad \text { or } \quad f_{2-\operatorname{dim}}=\frac{\mathscr{A} / a^{2}}{V / a^{4}}, \quad \text { etc. }
$$

where in the first, one-dimensional, case $\mathscr{L}$ is the total length of "localizing material" and lattice spacing $a$, or in the two-dimensional case, $\mathscr{A}$ is the area of two-dimensional material, and so on for higher dimensions. The (possibly fractal) dimension, $d$, of the localizing manifold is given by the scaling of the IPR as the lattice spacing is varied,

$$
I=1 / f \sim a^{d-4}
$$


In recent years a growing number of authors have found evidence for localization of either the LDE or other quantities, such as the topological charge density [2]. Since the appearance of [1]

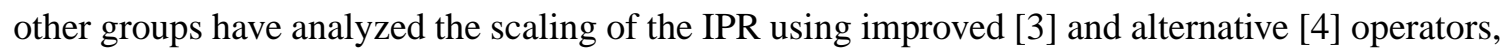
finding similar conclusions.

While the scaling of the IPR is a rather clean indication of the underlying localization dimension, its extraction requires a wide range of lattice spacings to get a fit with good statistics. In [1] for example, the dimension was given as somewhere between 2 and 3. For best results, scaling measurements should be done at fixed physical volume, which can then be compared to similar measurements at fixed lattice spacing to understand finite size issues.

\section{Scaling of the IPR}

In [1] we presented preliminary results for the scaling of the IPR using quenched lattices (Symanzik 1-loop improved gauge action) ranging from $12^{4}$ and $a=0.2 \mathrm{fm}$ up to $24^{4}$ and $a \sim 0.1$ $\mathrm{fm}$. However, with better statistics on the finest lattices, we found that the lattice spacing was closer to $a=0.095 \mathrm{fm}, 5 \%$ from of our target of $a=0.1 \mathrm{fm}$. In order to maintain a fixed volume we regenerated this ensemble at the target lattice spacing. Furthermore, as our lattice spacing is set using $r_{1}$ from the static quark potential, we use here an updated value of $r_{1}(0.317 \mathrm{fm})$ taken from [5] $\left(r_{1}=0.344 \mathrm{fm}\right.$ was used in [1] $)$. This increases all lattice spacings by $\sim 10 \%$, but has no effect on our results. Additionally, we have increased the convergence criteria for computing eigenvectors and added a finer 284 ensemble.

The lattices and parameters used for the present work are collected in the table below.

\begin{tabular}{|c|c|c|c|c|}
\hline$a$ & $L$ & vol & $\beta$ & no. configs. \\
\hline $0.218 \mathrm{fm}$ & 12 & $(2.61)^{4} \mathrm{fm}^{4}$ & 7.56 & 100 \\
0.163 & 16 & $(2.61)^{4}$ & 7.847 & 100 \\
0.128 & 20 & $(2.56)^{4}$ & 8.109 & 100 \\
0.110 & 24 & $(2.64)^{4}$ & 8.295 & 100 \\
0.0915 & 28 & $(2.56)^{4}$ & 8.527 & 100 \\
\hline
\end{tabular}

with 64 eigenvectors per lattice. The italicized ensemble was rerun for better convergence of eigenvectors (with very little change), while ensembles in teletype are new.

Our main result is summarized in Figure 1, where we plot the scaling of the average IPR using the lowest 8 eigenvectors. Compared with [- $[$ ] we have better statistics on the IPR values and, particularly, the new value at a smaller lattice spacing $(0.0915 \mathrm{fm})$ gives a much more precise scaling dimension for the IPR.

The best fit to the data has $d-4=0.934 \pm 0.149$ (see eq. 2.1), thus the scaling dimension, $d$, of the localization manifold is essentially 3 .

\section{Mobility Edge}

In analogy with the mechanism of Anderson localization in condensed matter physics, we can investigate whether our data shows a mobility edge, an energy above which quarks are delocalized and below which they show localization. This feature has been investigated by Golterman and 


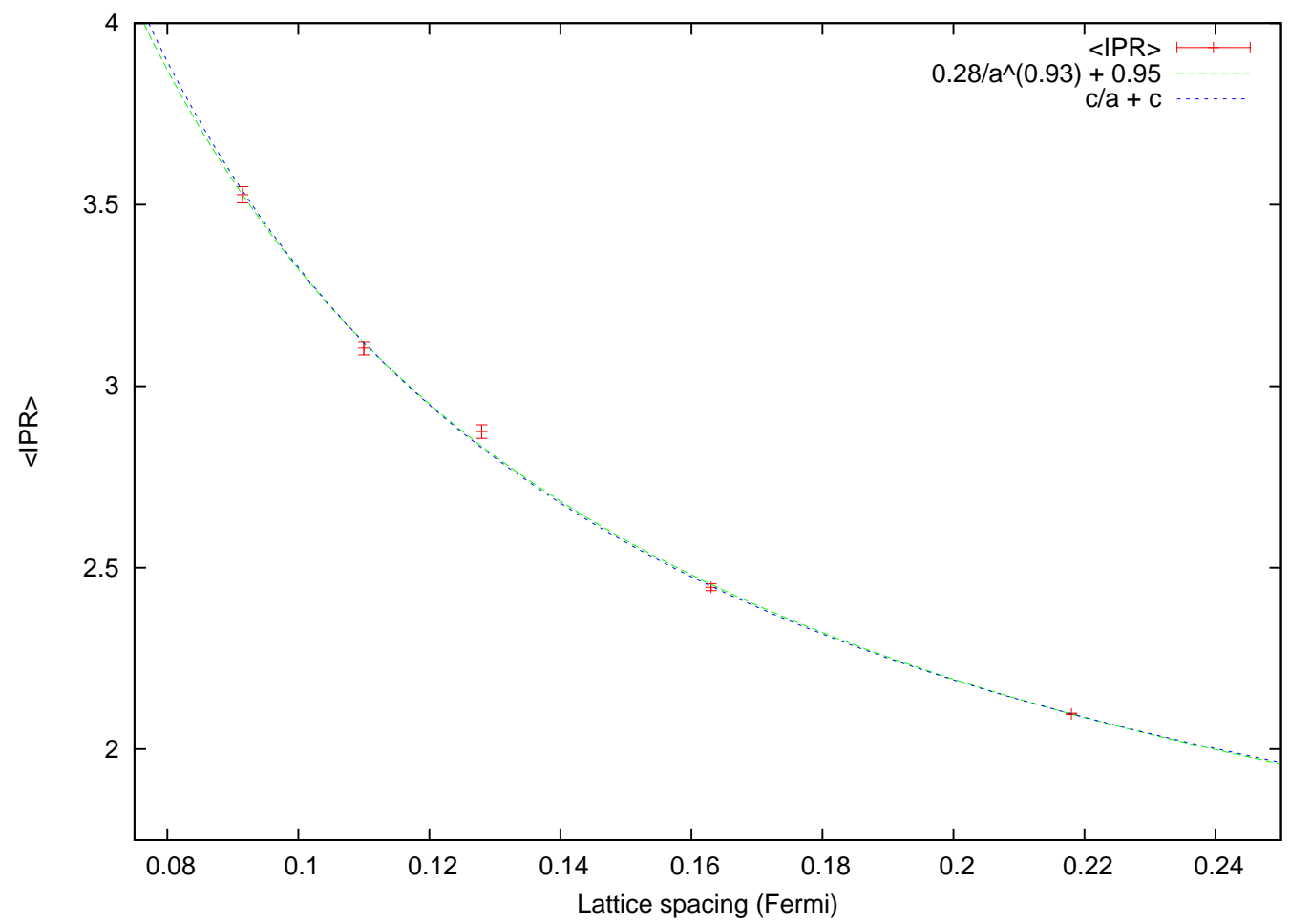

Figure 1: The scaling of the IPR for the lowest 8 eigenvectors, in red. In green is the best fit, and associated values. In blue is a fit to constant + constant $/ a$ for comparison.

Shamir [6] and observed in SU(2) [3] using an overlap Dirac operator with exact zero modes. The signal is a reduction in the IPR (less localization) at some critical value for the eigenvalue-the mobility edge.

In our SU(3) data the IPR values are rather low as compared to IPR values in SU(2) studies [3], [ 朋 (3-4 vs 5-20). One possible reason for this might be that the localization is due to topology of one of the $\mathrm{SU}(2)$ subgroups, while the other two subgroups randomize the eigenvectors. Whatever the reason, this is an interesting clue to understanding the localization.

Only our $28^{4}$ lattice at $a=0.0915 \mathrm{fm}$ shows a weak indication of the mobility edge, shown in Figure 2. Its value in physical units, around $50 \sim 100 \mathrm{MeV}$ is consistent with that seen in [3] . A study of the IPR on various volumes would be required to confirm this.

\section{Two-point Correlations}

While the IPR is a good quantitative indicator of localization, it only tells us the fraction of lattice sites where the eigenvector is large. If we rearranged the lattice sites we would obtain the same IPR. The two-point correlator on the other hand gives information on the connectedness of the eigenvector, and should drop off as $\sim r^{d-4}$ if the eigenvector is localized uniformly on a $d$ dimensional manifold.

We have computed the "all-to-all" correlator, $\langle\rho(x) \rho(y)\rangle$ and present the average at different spatial separation $|x-y|$ for eigenvalue 0 in Figure 3. These correlators have parity sawtooth 


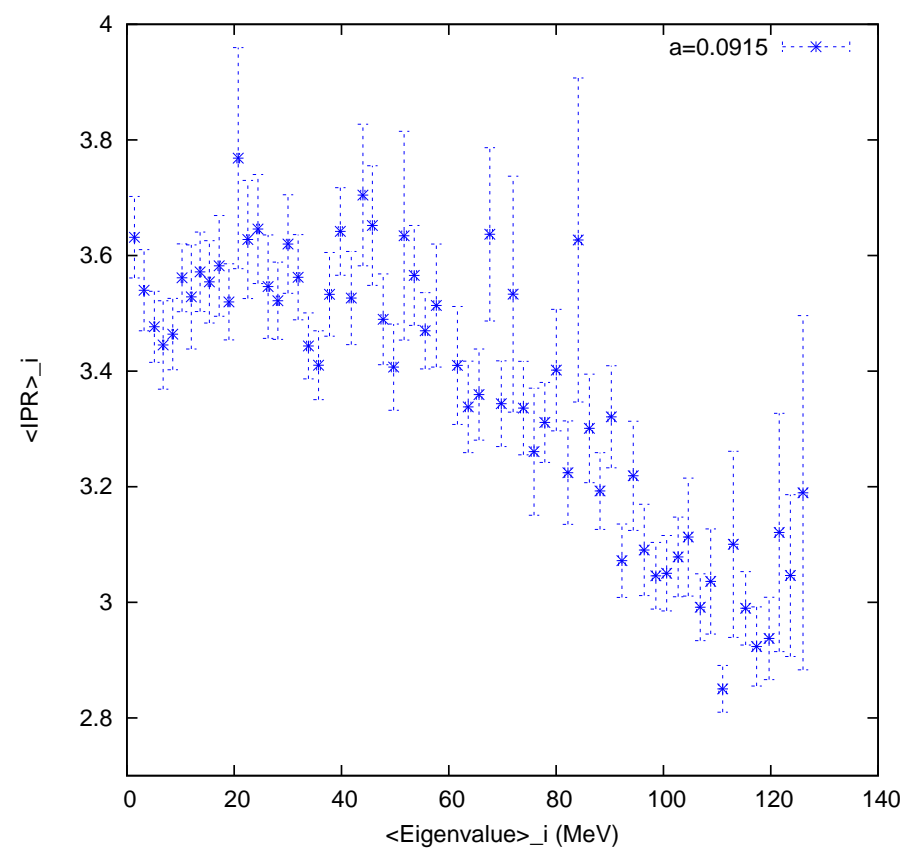

Figure 2: $\langle$ IPR $\rangle$ vs eigenvalue in physical units. The mobility edge is weakly indicated by a decrease in IPR at about $60-100 \mathrm{MeV}$.

behavior familiar in staggered fermion propagators, which lessens as the lattice spacing decreases. Taking the slope of the correlator from the even points, we show a line with $d=3.5$ (a very similar result if achieved for the odd points which lie slightly below the line). We see that in physical units, the correlation disappears by about 1 fermi, for all of our lattices.

\section{Conclusions}

We have extended our study of the IPR of the lowest eigenvectors of the asqtad Dirac operator computed on quenched background gauge fields. A number of conclusions are apparent.

- The IPR scaling $\sim 1 / a$ implies a dimension $d=3$ for the localizing manifold. The signal is quite a bit clearer than in our previous study.

- We see a weak mobility edge in our finest lattices (only) at 50 100 MeV. This is consistent with other work. The fact that our signal is weak is likely attributed to the lack of exact zero modes of our Dirac operator and possibly to the larger gauge group (SU(3) vs SU(2)).

- The two point correlator $\langle\rho(x) \rho(x+r)\rangle$ suggests a fractal dimension of $\sim 3.5$. It should be noted that we have not finished computing this quantity on all of our largest lattices (Figure 3 represents only about 8 of our $28^{4}$ dataset, however the correlator shows little fluctuation from lattice to lattice). In physical units the correlation disappears at $\sim 1 \mathrm{fm}$.

Our findings do not support the naive picture where the low-lying Dirac eigenmodes are localized on monopoles $(d=1)$ or vortices $(d=2)$. The relationship between topological excitations 


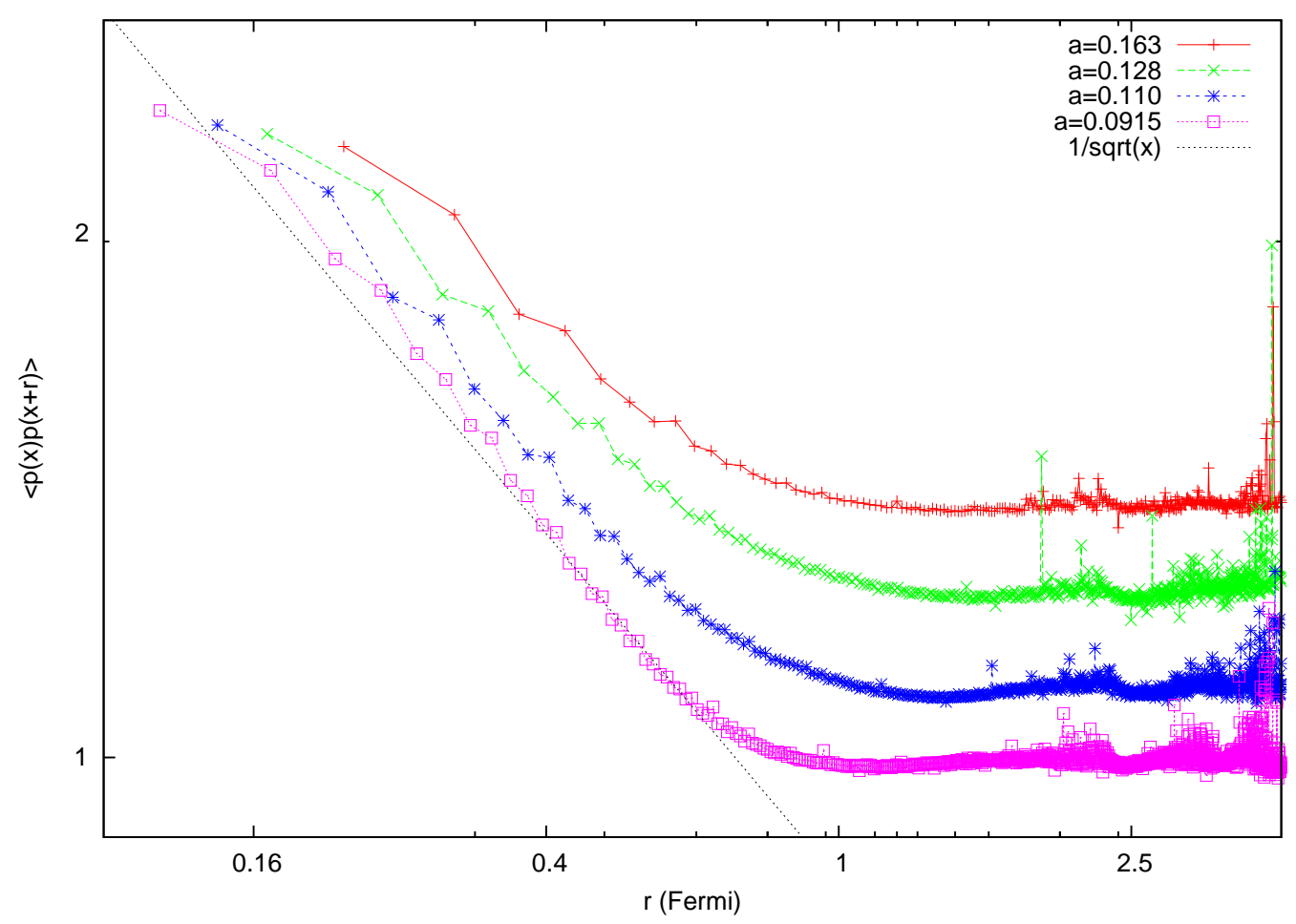

Figure 3: The two-point correlator of the lowest eigenvector, $\Sigma_{x}\langle\rho(x) \rho(x+r)\rangle$ as a function of distance $r$. Plots (except $\mathrm{a}=0.0915$ ) are displaced upward for clarity. The axes are log-log.

and eigenvector localization is more subtle. For instance, a set of center vortices gives eigenvectors localized weakly on the vortices themselves, and more strongly on their intersections [7].

\section{References}

[1] C. Aubin et al., The scaling dimension of low lying dirac eigenmodes and of the topological charge density, Nucl. Phys. (Proc.Suppl.) 140 (626) [hep-lat/ 0410024 ]

[2] I. Horvath et al. On the local structure of topological charge fluctuations in QCD, Phys. Rev. D67 (011501) [hep-lat/0203027]

[3] F.V. Gubarev et al., Evidence for fine tuning of fermionic modes in lattice gluodynamics [hep-lat/0505016]

[4] J. Greensite et al., Localized eigenmodes of covariant Laplacians in the Yang-Mills vacuum, Phys. Rev. D71 (114507) [hep-lat/ 0504008 ]

[5] C. Aubin et al., Light pseudoscalar decay constants, quark masses, and low energy constants from three-flavor lattice QCD, Phys. Rev. D70 (114501) [hep-lat/ 0407028 ]

[6] M. Golterman and Y. Shamir, Localization in lattice QCD, Phys. Rev. D68 (074501) [hep-lat/0306002]

[7] H. Reinhardt et al., Quark zero modes in intersecting vortex gauge fields, Phys Rev. 66 (085004) [hep-th/0203012] 